\title{
Effect of leptin on intestinal re-growth following massive small bowel resection in rat
}

Published online: 19 November 2005

(C) Springer-Verlag 2005

\begin{abstract}
Recent evidence suggests that the adipose tissue-derived cytokine leptin (LEP) is involved in modulation of growth and differentiation of normal small intestine. The purpose of the present study was to evaluate the effects of parenteral LEP on structural intestinal adaptation, cell proliferation and apoptosis in a rat model of short bowel syndrome (SBS). Male Sprague-Dawley rats were divided into three experimental groups: Sham rats underwent bowel transection and re-anastomosis, SBS-rats underwent a $75 \%$ small bowel resection, and SBS-LEP-rats underwent bowel resection and were treated with LEP given subcutaneously at a dose of $20 \mu \mathrm{g} / \mathrm{kg}$, once daily, from day 3 through 14. Parameters of intestinal adaptation (bowel and mucosal weights, mucosal DNA and protein, villus height and crypt depth in jejunum and ileum), enterocyte proliferation and enterocyte apoptosis were determined on day 15 following operation. Ileal tissue samples were taken for detection of bax and bcl-2 gene expression using RT-PCR technique. Statistical analysis was performed using the non-parametric Kruskal-Wallis ANOVA test, with $P<0.05$ considered statistically significant. Treatment with subcutaneous LEP resulted in a significant increase in jejunal $(17 \%, P<0.05)$ and ileal $(13 \%, P<0.05)$ bowel weight, jejunal $(10 \%, P<0.05)$ and ileal $(25 \%, P<0.05)$ mucosal weight, jejunal $(26 \%$, $P<0.05)$ and ileal $(38 \%, P<0.05)$ mucosal DNA, ileal
\end{abstract}

\footnotetext{
I. Sukhotnik $(\bowtie) \cdot$ Z. Vadasz $\cdot$ M. Lurie $\cdot$ O. A. Hatoum

J. G. Mogilner

Department of Pediatric Surgery B, Rappaport

Faculty of Medicine, Technion, Bnai Zion Medical Center, 47 Golomb Street, P.O.B. 4940, 31048 Haifa, Israel

E-mail: igor-dr@internet-zahav.net

Tel.: + 972-4-8256815

Fax: +972-4-8359620

E. Shiloni

Carmel Medical Center, Haifa, Israel
}

\section{A. G. Coran}

Section of Pediatric Surgery, Mott Children's Hospital, University of Michigan, Ann Arbor, MI, USA
$(25 \%, P<0.05)$ mucosal protein, jejunal $(41 \%, P<0.05)$ and ileal $(21 \%, P<0.05)$ villus height, jejunal $(37 \%$, $P<0.05)$ crypt depth, and jejunal $(24 \%, P<0.05)$ and ileal $(21 \%, P<0.05)$ enterocyte proliferation compared to SBS-animals. Enterocyte apoptosis increased significantly after bowel resection in jejunum and ileum compared to sham animals and was accompanied by an increased bax gene expression and a decreased bcl-2 gene expression in ileal samples. SBS-LEP rats showed a trend toward a decrease in enterocyte apoptosis in ileum and a mild decrease in bax gene expression compared to SBS-untreated animals. In conclusion, in a rat model of SBS parenteral LEP stimulates structural intestinal adaptation. Increased cell proliferation and decreased cell death via apoptosis may be responsible for this increased cell mass.

Keywords Short bowel syndrome $\cdot$ Intestinal adaptation - Leptin ' Enterocyte proliferation . Enterocyte apoptosis

\section{Introduction}

After massive small bowel resection, the remaining gut undergoes morphologic and functional changes to maintain normal absorptive capacity. Morphologic changes include enlargement of the remaining bowel, thickening of the intestinal wall, and an increase in villus height and microvillus surface area. Functional changes result in an enhanced nutrient uptake by isolated enterocytes [15]. A large number of nutrients, hormones and peptide growth factors are known to stimulate intestinal adaptation. The obese gene protein product leptin (LEP) is a hormone that is secreted from adipocytes and acts primarily on the hypothalamus regulating energy expenditure and food intake [4]. LEP is well known to be involved in the regulation of food intake and body weight. LEP is an attractive candidate for the treatment of obesity as it is an endogenous protein and has been demonstrated to have potent effects on body 
weight and adiposity in rodents [16]. Although intestine is not a classic target tissue for LEP, extensive studies in various experimental models have established that LEP determines important physiological effects on intestinal growth, cell maturation and differentiation $[1,8]$.

The purpose of this study was to evaluate the effects of LEP on structural intestinal adaptation following massive small bowel resection in rat and to determine the mechanisms by which LEP influences mucosal hyperplasia as well as its effect on enterocyte proliferation and cell death via apoptosis.

\section{Materials and methods}

\section{Animals}

This protocol was approved by the Animal Research Committee of Rappaport Faculty of Medicine, Technion (Haifa, Israel) in compliance with the guidelines established by the "Guide for the Care and Use of Laboratory Animals". Sprague-Dawley rats weighing 250-270 g were used for the experiment. The animals underwent a 4-day period of acclimatization under standardized conditions (12 h light-dark cycle, controlled room temperature) and were fed a standard rat chow.

\section{Study design}

Animals were divided randomly into three experimental groups of ten rats each. Group A rats underwent bowel transection and re-anastomosis (Sham), group B animals underwent $75 \%$ bowel resection [short bowel syndrome (SBS)], and group $\mathrm{C}$ underwent bowel resection and were treated with LEP given subcutaneously at a dose of $20 \mu \mathrm{g} / \mathrm{kg}$, once daily, from day 3 through 14 postoperatively (SBS-LEP).

\section{Operative technique}

Following an overnight fast, the animals were anaesthetized with an intraperitoneal injection of ketamine $(90 \mathrm{mg} / \mathrm{kg})$ and xylasine $(10 \mathrm{mg} / \mathrm{kg})$. The abdomen was opened through a midline incision. In resected animals, a point $5 \mathrm{~cm}$ from the ligament of Treitz as well as a point $10 \mathrm{~cm}$ proximal to the ileocecal junction were used to determine the extent of bowel resection. The remaining bowel was re-anastomosed using a single layer of interrupted 6-0 silk sutures, ensuring patency of the lumen. A sham group of animals underwent distal small bowel transection (at a point $10 \mathrm{~cm}$ proximal to the ileocecal junction) and re-anastomosis without resection. In all animals, the abdomen was closed with interrupted 3-0 Dexon sutures. Following surgery, the rats were fasted for $24 \mathrm{~h}$ and then were fed a standard chow diet ad lib.

\section{Parameters of adaptation}

Rats were sacrificed on day 15 following surgery with an overdose of pentobarbital. The proximal jejunum and distal ileum were quickly removed, rinsed with cold saline, and dried. Both segments were weighed, opened longitudinally, and the mucosa was scraped and weighed. Overall bowel and mucosal weight was calculated as milligram per centimeter of bowel length per $100 \mathrm{~g}$ body weight. Mucosal samples were homogenized with TRIzol reagent (Gibco BRL, USA). DNA and protein were extracted by the method of Chomczynski [9]. DNA concentration was recorded spectrophotometrically and calculated as microgram per centimeter of bowel length per $100 \mathrm{~g}$ body weight. Final protein concentration was measured spectrophotometrically using a commercially available kit (Bio-Rad, Protein Assay) and was calculated as microgram per centimeter of bowel length per $100 \mathrm{~g}$ body weight.

\section{Histologic examination}

After fixing in $4 \%$ buffered formalin, perpendicular sections from proximal jejunum and distal ileum were processed into standard paraffin blocks. Five micron tissue slices were stained with hematoxylin-eosin. These sections were studied microscopically using a micrometer eyepiece.

\section{Enterocyte proliferation and apoptosis}

Crypt cell proliferation was assessed using 5-bromodeoxyuridine (5-BrdU). Standard BrdU labeling. reagent (Zymed Laboratories, Inc, San Francisco, CA, USA) was injected intraperitoneally at a concentration of $1 \mathrm{ml} /$ $100 \mathrm{~g}$ body weight $2 \mathrm{~h}$ before sacrifice. Tissue slices were stained with a biotinylated monoclonal anti-BrdU antibody system provided in a kit form (Zymed Laboratories). An index of proliferation was determined as the ratio of crypt cells staining positively for BrdU per 10 crypts.

Additional $5 \mu \mathrm{m}$ thick sections were prepared to establish the degree of enterocyte apoptosis. Immunohistochemistry for Caspase-3 (Caspase-3 cleaved concentrated polyclonal antibody; dilution 1:100; Biocare Medical, Walnut Greek, CA, USA) was performed for identification of apoptotic cells using a combination of the streptovidin-biotin-peroxidase method and microwave antigen retrieval on formalin-fixed, paraffinembedded tissues according to the manufacturer's protocols. A qualified pathologist blinded to the source of intestinal tissue performed all measurements.

Expression of bax and bcl-2 genes was determined using RT-PCR analysis. Total RNA was extracted using TRIzol reagent as described by Chomczynski [9]. Mucosal mRNA was reverse transcribed into cDNA using a standard protocol. PCR was performed using a 
Perkin-Elmer Kit, with primers made using the cloned published sequence of bax and bcl-2 (Table 1). The PCR products were size fractionated on a $2 \%$ agarose gel stained with ethidium bromide.

\section{Results}

\section{Mucosal parameters}

Short bowel syndrome rats (group B) demonstrated a significant increase in bowel weight in jejunum $(4.5$-fold increase, $P<0.05)$ and ileum $(2.5$-fold increase, $P<0.05)$ (Fig. 1), mucosal weight in jejunum (4-fold increase, $P<0.05$ ) and ileum (2-fold increase, $P<0.05$; Fig. 1), increase in mucosal DNA content in jejunum (3.5-fold increase, $P<0.05)$ and ileum $(2.5$-fold increase, $P<0.05)$ (Fig. 2), and mucosal protein in jejunum (4-fold increase, $P<0.05)$ and ileum $(2.5$-fold increase, $P<0.05)$ (Fig. 2) compared to sham animals (group A).

Treatment with LEP (group C) resulted in an additional increase in bowel weight in jejunum $(17 \%$, $P<0.05)$ and ileum $(13 \%, P<0.05)$, mucosal weight in jejunum $(10 \%, P<0.05)$ and ileum $(25 \%, P<0.05)$ (Fig. 1), increase in mucosal DNA content in jejunum $(26 \%, P<0.05)$ and ileum $(38 \%, P<0.05)$, and mucosal protein in ileum $(25 \%, P<0.05)$ compared to SBS-untreated animals (group B) (Fig. 2).

\section{Histopathologic changes}

Short bowel syndrome-rats (group B) demonstrated a significant increase in jejunal (562 \pm 38 vs. $419 \pm 40 \mu \mathrm{m}$, $P<0.05)$ and ileal $(442 \pm 35$ vs. $290 \pm 33, P<0.05)$ villus height as well as jejunal $(209 \pm 24$ vs. $177 \pm 7 \mu \mathrm{m}$, $P<0.05)$ and ileal $(194 \pm 10$ vs. $163 \pm 8 \mu \mathrm{m}, P<0.05)$ crypt depth when compared to sham animals (group A) (Fig. 3).

Treatment with LEP (group C) was associated with an additional (compared to SBS animals) increase in villus height in jejunum $(41 \%, P<0.05)$ and ileum $(21 \%$, $P<0.05)$ as well as crypt depth in jejunum $(37 \%$, $P<0.05$ ) compared to SBS untreated animals (group B).

\section{Cellular proliferation and apoptosis}

A significant increase in enterocyte proliferation occurred in rats following massive bowel resection (group B vs. sham animals, group A) in both jejunum (52\%, $P<0.05)$ and ileum $(57 \%, P<0.05)$ (Table 2). SBS-rats treated with LEP (group C) demonstrated an additional increase in cell proliferation in jejunum $(24 \%, P<0.05)$ and ileum $(21 \%, P<0.05)$ compared to SBS-untreated animals (group B).

Evaluation of enterocyte apoptosis using immunohistochemistry for caspase-3 showed a significant increase in the number of apoptotic cells in jejunum (twofold, $P<0.05$ ) and ileum (twofold, $P<0.05$ ) in SBSrats (group B) compared to sham animals (group A). Administration of LEP (group C) did not change apoptotic index in jejunum but showed a trend toward a decrease in cell apoptosis in ileum compared to SBSuntreated animals (group B); however, this trend did not achieve statistical significance.

\section{RT-PCR for Bax and BCL-2}

Short bowel syndrome rats (group B) showed an increased intensity of the bands of bax and a decreased intensity of the bands of bcl-2 compared to sham animals (Fig. 4). Treatment with LEP did not change bcl-2 expression significantly, but resulted in a mild decrease in ileal bax gene expression that led to a mild net antiapoptotic effect in gut epithelium in vivo (Fig. 5).

\section{Discussion}

Short bowel syndrome is defined as the decrease in intestinal length following small bowel resection below the minimal amount necessary for normal nutrient absorption [5, 6]. Decreased absorptive surface area, impaired enterohepatic circulation with a subsequent decrease in bile acid pool, gastric acid hypersecretion leading to relative pancreatic insufficiency, and dysmotility disorders of the remaining gut with subsequent bacterial overgrowth lead to diarrhea, steathorrhea, nutrient and micronutrient malabsorption, vitamin deficiency, and impaired normal growth and development. SBS has significant morbidity and is potentially lethal especially when bowel loss is extensive [6].

Although intestinal transplantation has emerged as a feasible alternative in the treatment of children with SBS in the last two decades, intestinal adaptation remains the only chance for survival in a subset of these patients. Intestinal adaptation is defined as a process of progressive recovery from intestinal failure following bowel resection [15]. The remaining intestine dilates and elongates, and the villi become longer and the crypts deeper, leading to an increased absorptive surface area and an increased number of enterocytes. Functional adaptation results in the enhanced absorption of nutrients per unit intestinal length in vivo. The recruitment of the bulk of
Table 1 Oligonucleotide primers for RT-PCR detection of bax and bcl-2 mRNA transcripts

\begin{tabular}{lll}
\hline Genes & Sequence $5^{\prime}$ & Sequence $3^{\prime}$ \\
\hline Bax & ATGGACGGGTCCGGGGAGCA & ATGGACGGGTCCGGGGAGCA \\
Bcl-2 & TGAGGCCCTGTCTGCTTCTG & AGGCTCCCGGGGCAGTCATGA \\
\hline
\end{tabular}




\section{Macroscopic Bowel Appearance}

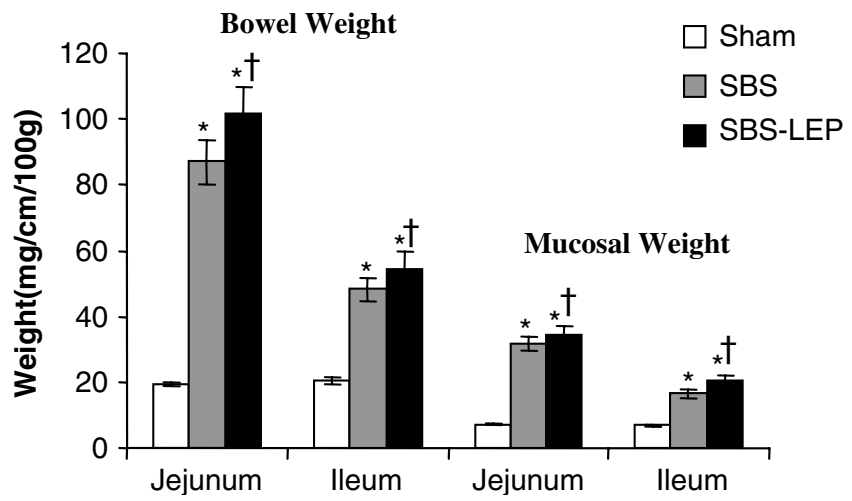

Fig. 1 Effect of bowel resection and parenteral leptin (LEP) on the macroscopic appearance of the remaining small intestine. Values are mean \pm SEM. SBS short bowel syndrome; LEP leptin. ${ }^{*} P<0.05$ SBS vs. Sham rats, $\uparrow P<0.05$ SBS-LEP vs. SBS rats

immature enterocytes due to the great hyperplastic stimulus seen following bowel resection appears to result in a reduction in the transport capacity of these cells [21]. On the other hand, nutrient uptake of mature enterocytes increases significantly through the increase in brush border membrane permeability and increased carrier-mediated transport [11]. There are a number of factors that can stimulate and accelerate intestinal adaptation. They include luminal nutrients, gastrointestinal secretions, hormones and peptide growth factors. Over the past three decades, an increasing number of regulatory peptides produced by cellular constituents have been identified.

Leptin is a nutritionally regulated adipocyte-derived $16-\mathrm{kDa}$ cytokine that was first discovered in 1994. Plasma LEP was found to highly correlate with body mass index (BMI) in rodents and in lean and obese humans [13]. Weight loss due to food restriction was associated with a decrease in plasma LEP in samples from mice and obese humans. LEP activates the long form of the LEP receptor to control feeding and neuroendocrine function and thus to regulate adiposity. While adiposity influences insulin sensitivity, LEP also regulates glucose homeostasis independent of energy

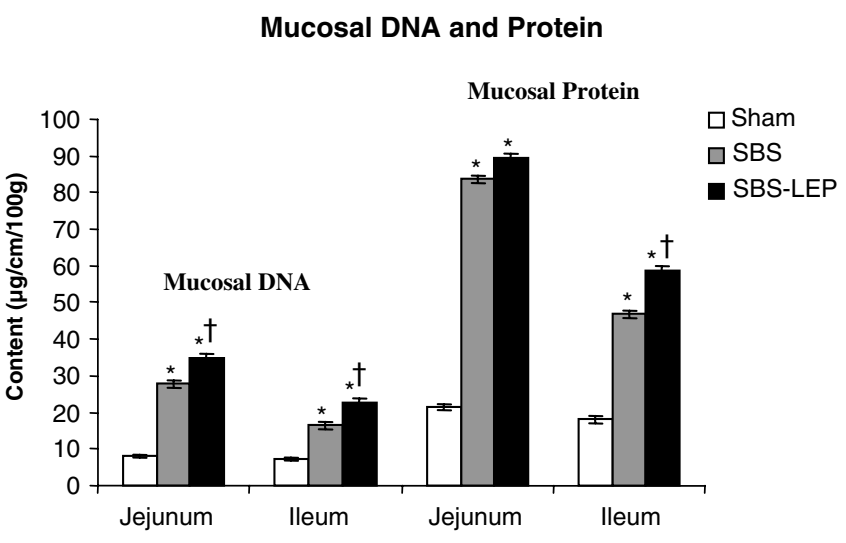

Fig. 2 Effect of bowel resection and treatment with LEP on mucosal DNA and protein content. Values are mean \pm SEM. $S B S$ short bowel syndrome; LEP leptin. ${ }^{*} P<0.05$ SBS vs. Sham rats, $\dagger$ $P<0.05$ SBS-LEP vs. SBS rats

balance [3]. LEP reflects not only the body fat level but also its nutritional status. Although LEP is a hormone mainly produced by the adipose tissue, the fact that LEP is present in human mature milk, that gastrointestinal

\section{Microscopic Bowel Appearance}

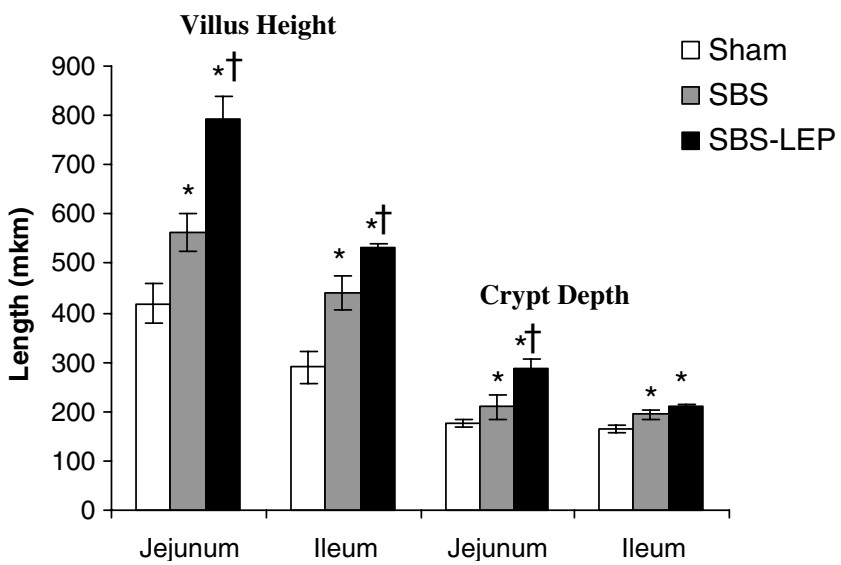

Fig. 3 Effect of bowel resection and treatment with LEP on the microscopic appearance of the remaining small intestine. Values are mean \pm SEM. SBS short bowel syndrome; $L E P$ leptin. ${ }^{*} P<0.05$ SBS vs. Sham rats, $\dagger P<0.05$ SBS-LEP vs. SBS rats

Table 2 Effect of bowel resection and treatment with leptin on enterocyte proliferation and apoptosis

\begin{tabular}{|c|c|c|c|}
\hline Parameters & Sham (group A, $n=10$ ) & SBS (group B, $n=10$ ) & SBS-LEP (group C, $n=10$ ) \\
\hline \multicolumn{4}{|c|}{$\begin{array}{l}\text { Enterocyte proliferation } \\
\text { (BrdU positive cells } 10 \text { crypts) }\end{array}$} \\
\hline Jejunum & $121 \pm 7$ & $184 \pm 21^{*}$ & $228 \pm 16^{*}, * *$ \\
\hline Ileum & $121 \pm 5$ & $190 \pm 20^{*}$ & $230 \pm 17 *, * *$ \\
\hline \multicolumn{4}{|c|}{$\begin{array}{l}\text { Enterocyte apoptosis } \\
\text { (Apoptotic cells/10 villi) }\end{array}$} \\
\hline Jejunum & $1.9 \pm 10.4$ & $3.7 \pm 0.5^{*}$ & $3.6 \pm 0.7^{*}$ \\
\hline Ileum & $1.8 \pm 10.4$ & $3.9 \pm 1.1^{*}$ & $2.2 \pm 0.4$ \\
\hline
\end{tabular}

Values are mean $\pm \mathrm{SEM}$

$S B S$ short bowel syndrome; $L E P$ leptin

$* P<0.05$ SBS vs. Sham rats; $* * P<0.05$ SBS-LEP vs. SBS rats 
Fig. 4 Effect of bowel resection and parenteral LEP on crypt cell proliferation and enterocyte apoptosis in jejunum and ileum. 5-BrdU incorporation into proliferating jejunal and ileal crypt cells was detected with a goat anti-BrdU antibody, and immunochemistry for caspase- 3 was used to determine enterocyte apoptosis
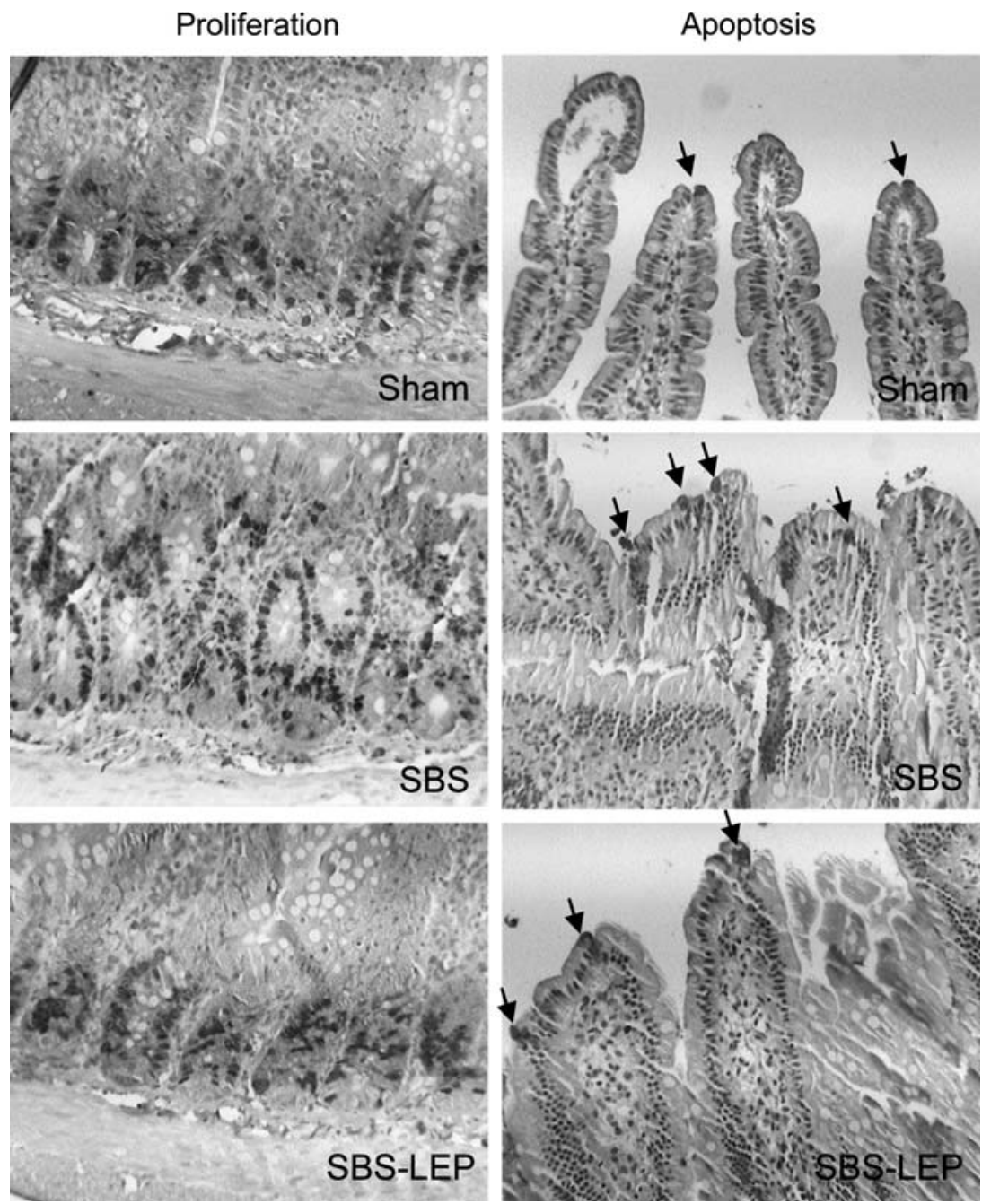

mucosa is capable of producing this growth factor [7] and that LEP receptors are expressed in both basolateral and brush border membranes of the enterocytes [2] suggests its potential role in small intestinal growth and development. It has been reported that the central nervous system, which regulates feeding behavior, might control intestinal function through the regulation of intestinal apoptosis, and LEP is considered a mediator

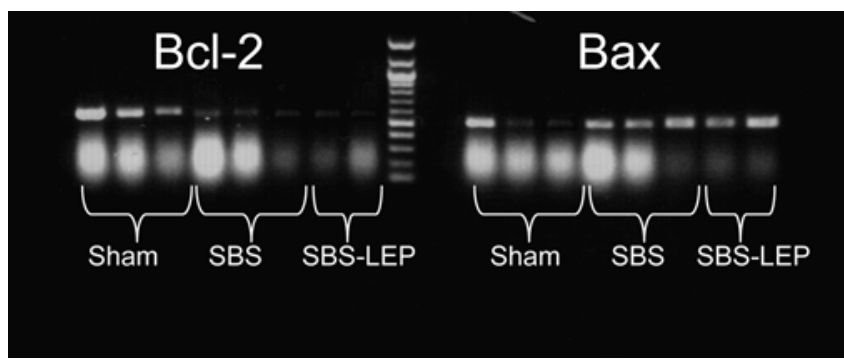

Fig. 5 Effect of bowel resection and treatment with LEP on expression of bax and bcl-2 in ileal mucosal samples. Intensity of the bands of bax is stronger and of bcl-2 is weaker in SBS-rats compared to sham animals. Treatment with LEP results in a mild decrease in the intensity of bands of bax compared with SBS animals between the neuroendocrine system and the intestine. Lin et al. [12] have recently shown that test solutions containing LEP induce jejunal mucosal apoptosis $3 \mathrm{~h}$ after infusion.

The effect of LEP on the growing remaining gut following massive small bowel resection is unclear. Pearson et al. [17] examined the effect of systemic LEP administration on small bowel absorptive function after massive small bowel resection in rats. They have shown that LEP enhances small intestine carbohydrate absorption beyond the normal adaptive response in a rat model of SBS. The authors concluded that LEP may be clinically useful in patients with inadequate intestinal function. In contrast, Chaudhary et al. [8] did not observe the stimulating effect of LEP on intestinal cell proliferation; however, they did observe a paradoxical inhibitory action on the caecum and colon.

In the present study, we evaluated the effect of parenteral LEP on structural intestinal adaptation in a rat model of SBS. Alterations in bowel and mucosal weights, mucosal DNA and protein content and histological appearance were used as indices of adaptation. Consistent with previous studies [18, 19], bowel resection in a rat results in apparent stimulation of structural 
intestinal adaptation. This is evident from increased bowel and mucosal weight, mucosal DNA and protein content, villus height and crypt depth. Increased bowel growth was found in both jejunum and ileum. Some authors have found this response only true in the small bowel segment distal to the site of anastomosis [14]. Although the muscular coat of the bowel may thicken slightly in the jejunal segment proximal to anastomotic site, the epithelial changes are more striking and account for most of the increase in bowel weight. 5-Bromodeoxyuridine incorporation was used to evaluate the kinetic changes that followed a massive small bowel resection. A significant increase in the number of proliferating cells was observed in both jejunum and ileum in SBS rats compared to sham animals and was accompanied by the augmented villus height and crypt depth, suggesting that the mucosal hyperplastic response is a result of increased cell proliferation and accelerated migration along the villus. Evaluation of enterocyte apoptosis in the present study was performed using immunohistochemistry for caspase- 3 and has shown increased cell apoptosis in both jejunum and ileum in rats with SBS. This observation is consistent with our previous reports and data from other investigators [10]. Increased apoptosis promotes disposal of genetically aberrant stem cells, prevents tumorogenesis and is considered as a mechanism that counterbalances the increased enterocyte proliferation seen in order to reach a new homeostatic set during intestinal adaptation. The observed pro-apoptotic effect of bowel resection was through regulation of expression of genes related to apoptosis. Apoptosis is a highly regulated and reproducible event that ultimately leads to cell death. Several regulatory genes affecting apoptosis have been identified and divided into pro-apoptotic genes (bax, bik, bak, bclxs, bad, p53, c-jun, hrk) and anti-apoptotic genes (bcl-2, bcl- $\mathrm{x}_{\mathrm{L}}, \mathrm{rb}, \mathrm{mcl}-1, \mathrm{a}$ 1, brag-1, bfl-1). Increased enterocyte apoptosis in the present study was accompanied by an enhanced bax gene expression and an inhibited bcl-2 gene expression compared to sham animals. This observation is consistent with data reported by other investigators [20].

Treatment with LEP had a strong stimulating effect on bowel re-growth following bowel resection in the rats. Parenteral administration of LEP led to an additional increase in bowel and mucosal weight in both jejunum and ileum compared to SBS-untreated animals. A concomitant increase in mucosal DNA and protein content suggests enhanced cell metabolism, which is consistent with the increased epithelial cell proliferation and differentiation. Not only were mucosal parameters of structural adaptation increased, but also villus height and crypt depth, suggesting increased absorptive surface area and increased proliferating activity in the crypts. The enterocyte proliferation index increased significantly following LEP administration and may be considered the main mechanism responsible for increased cell mass. We found that bcl-2 expression in ileal mucosa did not change significantly in LEP treated animals; however, bax expression decreased, and this was associated with decreased gut epithelial apoptosis in vivo. This observation is contrary to the recent report of Lin et al. [12] who showed a direct pro-apoptotic effect of LEP on enterocytes in normal intestine.

In conclusion, in a rat model of SBS treatment with LEP enhances structural intestinal adaptation. Increased enterocyte proliferation and decreased cell death via apoptosis may be responsible for this positive effect.

\section{References}

1. Badman M, Flier JS (2005) The gut and energy balance: visceral allies in the obesity wars. Science 307:1909-1914

2. Barrenetxe J, Villaro AC, Guembe L, Pascual I, Muñoz-Navas M, Barber A, Lostao MP (2002) Distribution of the long leptin receptor isoform in brush border, basolateral membrane, and cytoplasm of enterocytes. Gut 50:797-802

3. Bates SH, Kulkarni RN, Seifert M, Myers MG Jr (2005) Roles for leptin receptor/ STAT3-dependent and -independent signals in the regulation of glucose homeostasis. Cell Metab 1:169-178

4. Bell-Anderson KS, Bryson JM (2004) Leptin as a potential treatment for obesity: progress to date. Treat Endocrinol 3:1118

5. Biller JA (1987) Short bowel syndrome. In: Grand RI, Sutphen JL, Dietz WH (eds) Pediatric nutrition. theory and practice. Butterworth Publishers, Stoneham, pp 481-487

6. Booth IW, Lander AD (1998) Short bowel syndrome. Bailliere's Clin Gastroenterol 12:739-772

7. Cammisotto PG, Renaud C, Gingras D, Delvin E, Levy E, Bendayan M (2005) Endocrine and exocrine secretion of leptin by the gastric mucosa. J Histochem Cytochem 53(7):851-860

8. Chaudhary M, Mandir N, FitzGerald AJ, Howard JK, Lord GM, Ghatei MA, Bloom SR, Goodlad RA (2000) Starvation, leptin and epithelial cell proliferation in the gastrointestinal tract of the mouse. Digestion 61:223-229

9. Chomczynski P (1993) A reagent for the single-step simultaneous isolation of RNA, DNA and proteins from cell and tissue samples. Biotechniques 15:532-534

10. Helmrath MA, Erwin CR, Shin CE, Warner BW (1998) Enterocyte apoptosis is increased following small bowel resection. J Gastrointest Surg 2:44-49

11. Hinnes OJ, Bilchik AJ, Zinner MJ, Skotzko MJ, Moser AJ, McFadden DW, Ashley SW (1994) Adaptation of the Na/ glucose cotransporter following intestinal resection. J Surg Res $57: 22-27$

12. Lin T, Sakata H, Ootani A, Fujise T, Tsunada S, Amemori S, Danjo A, Yokoyama F, Sakata Y, Iwakiri R, Toda S, Fujimoto K (2005) Apoptosis in rat jejunal mucosa is regulated partly through the central nervous system, which controls feeding behavior. J Gastroenterol Hepatol 20:1285-1289

13. Nylander G, Olerud S (1962) Intestinal adaptation following extensive resection in the rat. Acta Chir Scand 123:51-56

14. Maffei M, Halaas J, Ravussin E, Pratley RE, Lee GH, Zhang Y, Fei H, Kim S, Lallone R, Ranganathan S (1995) Leptin levels in human and rodent: measurement of plasma leptin and ob RNA in obese and weight-reduced subjects. Nat Med 1:1155-1161

15. O'Brien DP, Nelson LA, Huang FS, Warner BW (2001) Intestinal adaptation: structure, function, and regulation. Sem Pediatr Surg 10:55-64

16. Paracchini V, Pedotti P, Taioli E (2005) Genetics of leptin and obesity: a HuGE review. Am J Epidemiol 162:101-114

17. Pearson PY, O’Connor DM, Schwartz MZ (2001) Novel effect of leptin on small intestine adaptation. Surg Res 97:192-195

18. Sukhotnik I, Yakirevich E, Coran AG, L.Siplovich, Hirsh M, Sabo E, Krausz M, Shiloni E (2002) Transforming growth factor-alpha increases enterocyte proliferation, decreases apoptosis and stimulates intestinal adaptation in a rat model of short bowel syndrome. J Surg Res 108:235-242 
19. Sukhotnik I, Mor-Vaknin N, Drongowski RA, Miselevich I, Coran AG, Harmon CM (2004) Effect of dietary fat on early morphological intestinal adaptation in a rat with short bowel syndrome. Pediatr Surg Int 20:419-424

20. Stern LE, Huang F, Kemp CJ, Falcone RA Jr, Erwin CR, Warner BW (2000) Bax is required for increased enterocyte apoptosis after massive small bowel resection. Surgery 128:165170

21. Whang EE, Dunn JC, Joffe H, Mahanty H, Zinner MJ, McFadden DW, Ashley SW (1996) Enterocyte functional adaptation following intestinal resection. J Surg Res 60:370374 